BNL-46459

AD/RHIC-102

Informal Report

\title{
Theory of the Beta Function Shift Due to Linear Coupling
}

\author{
G. Parzen
}

July 1991

\section{R H I C P R O J E C T}

\author{
Brookhaven National Laboratory \\ Associated Universities, Inc. \\ Upton, NY 11973
}




\title{
THEORY OF THE BETA FUNCTION SHIFT DUE TO LINEAR COUPLING
}

\author{
G. Parzen \\ Brookhaven National Laboratory \\ July 1991
}

\section{Introduction}

This paper presents analytical perturbation theory results for $\beta_{1}, \beta_{2}$, the beta functions in the presence of linear coupling. It is a continuation of a previous paper ${ }^{1}$ that gave analytical perturbation theory results for the tune $\nu_{1}, \nu_{2}$ in the presence of linear coupling. The results for $\beta_{1}, \beta_{2}$ hold when $\nu_{x}, \nu_{y}$ are close to the resonance line $\nu_{x}-\nu_{y}=p$. The shift in beta functions is then linear in the skew quadrupole field given by $a_{1}(s)$. When $\nu_{x}, \nu_{y}$ are far enough from the $\nu_{x}-\nu_{y}=p$ resonance, then the shift in the beta function becomes quadratic in the skew quadrupole field.

The analytical results show that the important harmonics in the skew quadrupole fields for producing large beta functions shifts are the harmonics near $\nu_{x}+\nu_{y}$. The harmonics near $\nu_{x}+\nu_{y}$ are also the important harmonics for the higher order tune (see Ref. 1). It is also shown that the beta function shift and the higher order tune shift have the same driving terms, thus, one may expect that an $a_{1}$ correction system that corrects the higher order tune shift will also correct the beta function shift. 


\section{Lowest Order Solution for $\beta_{1}$ and $\beta_{2}$}

The presence of the skew quadrupole fields will couple the $x$ and $y$ motions. New beta functions, $\beta_{1}, \beta_{2}$ can be defined ${ }^{2}$ which are the beta functions of the normal modes and which are different from $\beta_{x}, \beta_{y}$, the beta functions of the unperturbed accelerator.

It will be shown below that $\beta_{1}$ and $\beta_{2}$ can be found from the solutions of the equations of motions, Eq. (2.1) in reference 1. These solutions were written there as

$$
\begin{aligned}
\eta_{x} & =\zeta_{x}+\zeta_{x}^{*}, \quad \eta_{y}=\zeta_{y}+\zeta_{y}^{*} \\
\zeta_{x} & =A_{s} \exp \left(i \nu_{x, s} \theta_{x}\right)+\sum_{r \neq s} A_{r} \exp \left(i \nu_{x, r} \theta_{x}\right), \\
\zeta_{y} & =B_{s} \exp \left(i \nu_{y, s} \theta_{x}\right)+\sum_{r \neq s} B_{r} \exp \left(i \nu_{y, r} \theta_{y}\right), \\
\nu_{x, s}-\nu_{y, s} & =p
\end{aligned}
$$

$\zeta_{x}^{*}$ is the complex conjugate of $\zeta_{x}$.

The lowest order solution for the $A_{r}, B_{r}$ are given by Eq. (2.7) Ref. 1, which can be put into Eq. (2.1) to find $\zeta_{x}, \zeta_{y}$. The first two equations in Eq. (2.7), Ref. 1 show that the two large coefficients $A_{s}, B_{s}$ are related. For the $\nu_{1}$ mode, where $\nu_{1} \rightarrow \nu_{x}$ when $a_{1} \rightarrow 0$, and using $\nu_{x, s} \simeq \nu_{x}$ one finds

$$
B_{s}=\frac{-\left(\nu_{1}-\nu_{x}\right)}{\Delta \nu\left(\nu_{x, s}, \nu_{y, s}\right)} A_{s} .
$$

For the $\nu_{2}$ mode, using $\nu_{y, s} \simeq \nu_{y}$, one finds

$$
A_{s}=\frac{-\left(\nu_{2}-\nu_{y}\right)}{\Delta \nu^{*}\left(\nu_{x, s}, \nu_{y, s}\right)} B_{s} .
$$

$\Delta \nu\left(\nu_{x, s}, \nu_{y, s}\right)$ is defined by Eq. (2.8), Ref. 1 .

The last two equations of Eq. (2.7), Ref. 1, can be solved for $A_{r}$ and $B_{r}$, which can then be put into Eq. (2.1) to find the Floquet solutions. Note that $A_{r} \neq 0$ only for $\nu_{x, y}=\nu_{y, s}+n, n \neq p$, and $B_{r} \neq 0$ only for $\nu_{y, r}=\nu_{x, s}+n, n \neq-p$. Assuming that $\nu_{x}, \nu_{y}$ is close to the resonance line $\nu_{x, s}=\nu_{y, s}+p$, so that $\nu_{x, s} \simeq \nu_{x}$, and $\nu_{y, s} \simeq \nu_{y}$, then

$$
\begin{aligned}
& A_{r}=\frac{-2 \nu_{x} b_{x}\left(\nu_{x, r}, \nu_{y, s}\right)}{\left(n+\nu_{y, s}\right)^{2}-\nu_{x}^{2}} B_{s} \\
& A_{r}=\frac{-2 \nu_{x} b_{x}\left(\nu_{x, r}, \nu_{y, s}\right)}{\left(n+\nu_{x}+\nu_{y}\right)(n-p)} B_{s}
\end{aligned}
$$


where $n \neq p, \nu_{x, r}=\nu_{y, s}+n$ and $b_{x}$ is defined by Eq. (2.6), Ref. 1 .

Similarly, one finds for $B_{r}$

$$
\begin{aligned}
& B_{r}=\frac{-2 \nu_{y} b_{y}\left(\nu_{y, r}, \nu_{x, s}\right)}{\left(n+\nu_{x, s}\right)^{2}-\nu_{y}^{2}} A_{s} \\
& B_{r}=\frac{-2 \nu_{y} b_{y}\left(\nu_{y, r}, \nu_{x, s}\right)}{\left(n+\nu_{x}+\nu_{y}\right)(n+p)} A_{s}
\end{aligned}
$$

where $n \neq-p, \nu_{y, r}=\nu_{x, s}+n$

We can now find $\zeta_{x}$ for the $\nu_{1}$ mode using Eqs. (2.3) and (2.2a) for $A_{r}$ and putting these results into Eq. (2.1) for $\zeta_{x}$,

$$
\begin{aligned}
& \zeta_{x}=A_{s} e^{i \nu_{1} \theta_{x}}\left\{1+\sum_{n \neq-p} f_{n}\right\} \\
& f_{n}=\frac{\nu_{1}-\nu_{x}}{\Delta \nu\left(\nu_{x, s}, \nu_{y, s}\right)} \frac{2 \nu_{x} b_{n} \exp \left[-i(n+p) \theta_{x}\right]}{\left(n-\nu_{x}-\nu_{y}\right)(n+p)} \\
& b_{n}=\frac{1}{4 \pi \rho} \int d s a_{1}\left(\beta_{x} \beta_{y}\right)^{\frac{1}{2}} \exp \left[i\left(\left(n-\nu_{y}\right) \theta_{x}+\nu_{y} \theta_{y}\right)\right]
\end{aligned}
$$

A similar result can be found for $\zeta_{y}$ for the $\nu_{2}$ mode

$$
\begin{aligned}
& \zeta_{y}=B_{s} e^{i \nu_{2} \theta_{y}}\left\{1+\sum_{n \neq p} g_{n}\right\} \\
& g_{n}=\frac{\nu_{2}-\nu_{y}}{\Delta \nu^{*}\left(\nu_{x, s}, \nu_{y, s}\right)} \frac{2 \nu_{y} c_{n} \exp \left[-i(n-p) \theta_{y}\right]}{\left(n-\nu_{x}-\nu_{y}\right)(n-p)} \\
& c_{n}=\frac{1}{4 \pi \rho} \int d s a_{1}\left(\beta_{x} \beta_{y}\right)^{\frac{1}{2}} \exp \left[i\left(\left(n-\nu_{x}\right) \theta_{y}+\nu_{x} \theta_{x}\right)\right]
\end{aligned}
$$

From the above Floquet solutions for $\zeta_{x}, \zeta_{y}$, one can find $\beta_{1}$ and $\beta_{2}$ the beta functions of the normal modes. This will be done below. It may be noted that $b_{n}$ and $c_{n}$ are just the integrals involved in computing the stopbands of $\nu_{x}+\nu_{y}=n$ sum resonance, but at certain choices of the $\nu$-values on the resonance line. The $b_{n}$ corresponds to the tune choice $n-\nu_{y}, \nu_{y}$, and $c_{n}$ to $\nu_{x}, n-\nu_{x}$. The resonance denominator $1 /\left(n-\nu_{x}-\nu_{y}\right)$ shows that the important $n$ is near $\nu_{x}+\nu_{y}$.

The $x$ motion given by $x=\beta_{x}^{1 / 2}\left(\zeta_{x}+\zeta_{x}^{*}\right)$ is the $x$-motion when only the $\nu_{1}$ mode is excited. Similarly, $y=\beta_{y}^{1 / 2}\left(\zeta_{y}+\zeta_{y}^{*}\right)$ is the $y$ motion when only the $\nu_{2}$ mode is excited. 


\section{Results for $\beta_{1}$ and $\beta_{2}$}

It was shown by Edwards and Teng ${ }^{2}$ that one can transform from the $x, x^{\prime}, y, y^{\prime}$ coordinates to a new set of coordinates $v, v^{\prime} u, u^{\prime}$ which are uncoupled. The solutions of equations of motions for $u$ and $v$ can be written as ${ }^{2}$

$$
\begin{aligned}
& v=\sqrt{\beta_{1} \epsilon_{1}} \exp \left(i \psi_{1}\right)+\text { c.c. } \\
& u=\sqrt{\beta_{2} \epsilon_{2}} \exp \left(i \psi_{2}\right)+\text { c.c. }
\end{aligned}
$$

$\beta_{1}$ and $\beta_{2}$ are periodic functions and are the beta functions in the presence of linear coupling. If no solenoids are present, the $\psi_{1}$ and $\psi_{2}$ are related to $\beta_{1}, \beta_{2}$ by $^{2}$

$$
\begin{aligned}
& 1 / \beta_{1}=d \psi_{1} / d s \\
& 1 / \beta_{2}=d \psi_{2} / d s
\end{aligned}
$$

$\epsilon_{1}$ and $\epsilon_{2}$ are two constants that turn out to be the emittances of each normal mode.

The $x, x^{\prime}, y, y^{\prime}$ and the $v, v^{\prime}, u, u^{\prime}$ coordinates are related by ${ }^{2}$

$$
x=R v
$$

where $R$ is a $4 \times 4$ matrix given by

$$
R=\left(\begin{array}{cc}
I \cos \varphi & D \sin \varphi \\
-\bar{D} \sin \varphi & I \cos \varphi
\end{array}\right)
$$

$D$ and $\bar{D}$ are $2 \times 2$ matrices, and $\bar{D}=D^{-1}$. $I$ is the $2 \times 2$ identity matrix. $D$ and $\varphi$ can be computed from the one turn transfer matrix. ${ }^{2}$

Let $v, v^{\prime}$ be the coordinates that have the tune $\nu_{1}$ where $\nu_{1} \rightarrow \nu_{x}$ when $a_{1} \rightarrow 0$. Then if only this mode is present then $x$ is given by

$$
x=\cos \varphi v
$$

From Eq. (2.11) one finds

$$
\cos \varphi \sqrt{\beta_{1} \epsilon_{1}} \exp \left(i \psi_{1}\right)=\sqrt{\beta_{x}} \zeta_{x}
$$

where $\zeta_{x}$ is given by Eq. (2.5). It follows that

$$
\begin{aligned}
& \beta_{1}=d \psi / d s \\
& \zeta_{x}=\left|\zeta_{x}\right| \exp (i \psi) .
\end{aligned}
$$


and $\psi$ can be found from $\mathrm{Eq} .(2.5)$.

$$
\begin{aligned}
& \zeta_{x}=A_{s} \exp \left(i \nu_{1} \theta_{x}\right)\left(1+\sum_{n \neq-p} f_{n}\right) \\
& \zeta_{x}=A_{s}\left(1+\frac{1}{2} \sum_{n \neq-p}\left(f_{n}+f_{n}^{*}\right)\right) \exp \left[i\left(\nu_{1} \theta_{x}+\frac{1}{2 i} \sum_{n \neq-p}\left(f_{n}-f_{n}^{*}\right)\right)\right] \\
& \psi=\nu_{1} \theta_{x}+\frac{1}{2 i} \sum_{n \neq-p}\left(f_{n}-f_{n}^{*}\right) \\
& \frac{1}{\beta_{1}}=\frac{d \psi}{d s}=\frac{\nu_{1}}{\nu_{x} \beta_{x}}+\frac{1}{2 \nu_{x} \beta_{x}} \sum_{n \neq-p}(-n-p)\left(f_{n}+f_{n}^{*}\right) .
\end{aligned}
$$

Using $1 / \beta_{1}-1 / \beta_{x} \simeq-\left(\beta_{1}-\beta_{x}\right) / \beta_{x}^{2}$ one finds

$$
\begin{aligned}
& \frac{\beta_{1}-\beta_{x}}{\beta_{x}}=-\frac{\nu_{1}-\nu_{x}}{\nu_{x}}-\sum_{n \neq-p} \frac{(-n-p)}{2 \nu_{x}}\left(f_{n}+f_{n}^{*}\right) . \\
& \frac{\beta_{1}-\beta_{x}}{\beta_{x}}=-\sum_{\text {all } n}\left\{\frac{\nu_{1}-\nu_{x}}{\Delta \nu\left(\nu_{1}, \nu_{1}-p\right)} \frac{b_{n}}{n-\nu_{x}-\nu_{y}} \exp \left[-i(n+p) \theta_{x}\right]+\text { c.c. }\right\} .
\end{aligned}
$$

In a similar way, one also finds

$$
\frac{\beta_{2}-\beta_{y}}{\beta_{y}}=-\sum_{\text {all } n}\left\{\frac{\nu_{2}-\nu_{y}}{\Delta \nu^{*}\left(\nu_{2}+p, \nu_{2}\right)} \frac{c_{n}}{n-\nu_{x}-\nu_{y}} \exp \left[-i(n-p) \theta_{y}\right]+\text { c.c. }\right\} \text {. }
$$

Eq. (2.16) can be written in an integral form by using the result

$$
\sum_{\text {all } n} \frac{\exp \left[i n\left(\theta-\theta^{\prime}\right)\right]}{n-\nu}=-\pi \frac{\exp (\mp i \pi \nu)}{\sin \pi \nu} e^{i \nu\left(\theta-\theta^{\prime}\right)}
$$

where the top sign is used for $\theta>\theta^{\prime}$, and the bottom sign for $\theta<\theta^{\prime}$. Replacing $b_{n}$ using Eq. (2.5) one finds

$$
\begin{aligned}
\frac{\beta_{1}-\beta_{x}}{\beta_{x}} & =-\frac{\left(\nu_{1}-\nu_{x}\right)}{\left|\Delta \nu\left(\nu_{1}, \nu_{1}-p\right)\right|} \frac{1}{2 \rho \sin \pi\left(\nu_{x}+\nu_{y}\right)} \\
& \times \int d s^{\prime} a_{1}\left(s^{\prime}\right)\left(\beta_{x}\left(s^{\prime}\right) \beta_{y}\left(s^{\prime}\right)\right)^{\frac{1}{2}} \\
\cos \left[ \pm \pi\left(\nu_{x}+\nu_{y}\right)-\left(\nu_{x}+\nu_{y}\right)\left(\theta_{x}-\theta_{x}^{\prime}\right)\right. & \left.+\nu_{y}\left(\theta_{y}^{\prime}-\theta_{x}^{\prime}\right)-p \theta_{x}-\delta_{1}\right] .
\end{aligned}
$$


$\delta_{1}=$ phase $\left[\Delta \nu\left(\nu_{1}, \nu_{1}-p\right)\right]$, and in the \pm sign, the + sign is used for $\theta>\theta^{\prime}$, and the sign for $\theta<\theta^{\prime}$.

In a similar way one can find $\left(\beta_{2}-\beta_{y}\right) / \beta_{y}$ as

$$
\begin{array}{r}
\frac{\beta_{2}-\beta_{y}}{\beta_{y}}=-\frac{\left(\nu_{2}-\nu_{y}\right)}{\left|\Delta \nu\left(\nu_{2}+p, \nu_{2}\right)\right|} \frac{1}{2 \rho \sin \pi\left(\nu_{x}+\nu_{y}\right)} \\
\times \int d s^{\prime} a_{1}\left(s^{\prime}\right)\left(\beta_{x}\left(s^{\prime}\right) \beta_{y}\left(s^{\prime}\right)\right)^{\frac{1}{2}} \\
\cos \left[ \pm \pi\left(\nu_{x}+\nu_{y}\right)-\left(\nu_{x}+\nu_{y}\right)\left(\theta_{y}-\theta_{y}^{\prime}\right)\right. \\
\left.+\nu_{x}\left(\theta_{x}^{\prime}-\theta_{y}^{\prime}\right)+p \theta_{y}+\delta_{2}\right] .
\end{array}
$$

$\delta_{2}=$ phase $\left[\Delta \nu\left(\nu_{2}+p, \nu_{2}\right)\right]$.

Eq. (2.16) shows that the important harmonics in $a_{1}$ are the harmonics near $\nu_{x}+\nu_{y}$. However, Eq. (2.16) shows that the dominant harmonic excited in $\beta_{1}$ due to the $a_{1}$ field is the $2 \nu_{x}$ harmonic, and in $\beta_{2}$ the $2 \nu_{y}$ harmonic.

One may note the factor $\left(\nu_{1}-\nu_{x}\right) / \Delta \nu$. Close to the resonance line $\nu_{x}=\nu_{y}+p$ where $|\Delta \nu|>>\left|\nu_{x}-\nu_{y}-\nu_{p}\right|$, then this factor approaches 1. This may be seen from Eq. (2.10) in Ref. 1 for $\nu_{1}$ and $\nu_{2}$. According to Eq. (2.10), Ref. $1,\left(\nu_{1}-\nu_{x}\right) /|\Delta \nu| \rightarrow 1$ for large $\Delta \nu$, and $\left(\nu_{1}-\nu_{x}\right) /|\Delta \nu| \sim 2|\Delta \nu| /\left|\nu_{x}-\nu_{y}\right|$ for small enough $\Delta \nu$. Thus $\left(\beta_{1}-\beta_{x}\right) / \beta_{x}$ is linear in $a_{1}$ for large enough $\Delta \nu, \nu_{x}, \nu_{y}$ close enough to the resonance line, and quadratic in $a_{1}$ for small enough $\Delta \nu$, far enough from the resonance line. For small enough $\Delta \nu$ where $\left(\beta_{1}-\beta_{x}\right) / \beta_{x}$ becomes quadratic in $a_{1}$, then Eq. (2.16) is no longer correct because of the neglect of $a_{1}^{2}$ terms in deriving it.

A result for the rms value of $\left(\beta_{1}-\beta_{x}\right) / \beta_{x}$ due to a random distribution of $a_{1}$ errors may be obtained from the integral form Eq. (2.19), for the case when $|\Delta \nu|>>\left|\nu_{x}-\nu_{y}-p\right|$. In this case $\left|\nu_{1}-\nu_{x}\right| /|\Delta \nu| \simeq 1$ and

$$
\begin{aligned}
& \left(\frac{\beta_{1}-\beta_{x}}{\beta_{x}}\right)_{r m s}^{2}=\sum_{k}\left(\frac{\beta_{1}-\beta_{x}}{\beta_{x}}\right)_{k, r m s}^{2} \\
& \left(\frac{\beta_{1}-\beta_{x}}{\beta_{x}}\right)_{k, r m s}=N_{k}^{1 / 2} \frac{\left(\left(\beta_{x} \beta_{y}\right)^{1 / 2} a_{1, r m s}\right)_{k}}{2.8 \rho \sin \pi\left(\nu_{x}+\nu_{y}\right)}
\end{aligned}
$$

where the index $k$ indicates the different types of magnets. $N_{k}$ is the number of magnets of a certain type. Eq. (2.19) also gives the result for $\left(\left(\beta_{2}-\beta_{y}\right) / \beta_{y}\right)_{r m s}$. One also sees 
that

$$
\left(\left(\beta_{1}-\beta_{x}\right) / \beta_{x}\right)_{r m s}=\left[4 \pi /\left(2.8 \sin \pi\left(\nu_{x}+\nu_{y}\right)\right)\right] \Delta \nu_{r m s}
$$

where $\Delta \nu_{r m s}$ is the rms value of $\Delta \nu$.

\section{Correction of $\beta_{1}, \beta_{2}$}

The above analytical results for the beta function shifts show that when the higher order tune shifts $\nu_{1}-\nu_{x}$ and $\nu_{2}-\nu_{y}$ are corrected, then the beta function shifts are also corrected. This can be seen by comparing Eq. (2.5) for the beta function shift with Eq. (3.2 and 3.3) in Ref. 1, for the higher order tune shift. Both these effects have the same driving terms $b_{n}$ and $c_{n}$, and for both effects the important $b_{n}, c_{n}$ are those for which $n$ is close to $\nu_{x}+\nu_{y}$.

This result has been observed in numerical computations ${ }^{3}$ for the RHIC accelerator, where an $a_{1}$ correction system has been provided to correct the higher order tune shift. ${ }^{4,5}$ In order to correct the shift in the beta functions it is important that in correcting the higher order tune shift, that one correct not only the tune splitting $\left|\nu_{1}-\nu_{2}\right|$ but also the shift in the average tune $\left(\nu_{1}+\nu_{2}\right) / 2$. The harmonic closest to $\nu_{x}+\nu_{y}$ do not have much effect on $\left|\nu_{1}-\nu_{2}\right|$ but are most important for the average tune $\left(\nu_{1}+\nu_{2}\right) / 2$, and also for the beta function shift. One might be able to correct the average tune $\left(\nu_{1}+\nu_{2}\right) / 2$ using the normal tune adjusting quadrupoles instead of the $a_{1}$ correctors, but this would not help to correct the beta function shift.

\section{References}

1. G. Parzen, Theory of the Tune Shift due to Linear Coupling, BNL Report, AD/RHIC100, (1991).

2. D. Edwards and L. Teng, IEEE 1973 PAC, p. 885 (1973).

3. G. Parzen, IEEE 1991 PAC, to be published and BNL Report AD/RHIC-92, (1991).

4. G. Parzen, IEEE 1991 PAC, to be published and BNL Report AD/RHIC-93, (1991).

5. G. Parzen, BNL Report, AD/RHIC/AP-93 (1991). 